OPEN ACCESS

Edited by:

Julian O'Kelly,

Royal Hospital for Neuro-disability, UK

Reviewed by:

Pamela Heaton,

Goldsmiths University of London, UK

Kate Simpson,

Griffith University, Australia

*Correspondence:

Nandini C. Singh,

National Brain Research Centre, NH 8, Manesar, Gurgaon 122 050,

Haryana, India

nandini@nbrc.ac.in

Received: 01 April 2015 Accepted: 22 September 2015

Published: 29 October 2015

Citation:

Paul A, Sharda M, Menon S, Arora I, Kansal N, Arora K and Singh NC (2015) The effect of sung speech on socio-communicative responsiveness

in children with autism spectrum disorders.

Front. Hum. Neurosci. 9:555 doi: 10.3389/fnhum.2015.00555

\section{The effect of sung speech on socio-communicative responsiveness in children with autism spectrum disorders}

\author{
Arkoprovo Paul ${ }^{1}$, Megha Sharda ${ }^{1,2}$, Soumini Menon ${ }^{3}$, Iti Arora $^{3}$, Nayantara Kansal ${ }^{3}$, \\ Kavita Arora $^{3}$ and Nandini C. Singh ${ }^{1 *}$ \\ ${ }^{1}$ National Brain Research Centre, Gurgaon, India, ${ }^{2}$ International Laboratory of Brain, Music and Sound Research (BRAMS), \\ University of Montreal, Montreal, QC, Canada, ${ }^{3}$ Children First Mental Health Institute, New Delhi, India
}

There is emerging evidence to demonstrate the efficacy of music-based interventions for improving social functioning in children with Autism Spectrum Disorders (ASD). While this evidence lends some support in favor of using song over spoken directives in facilitating engagement and receptive intervention in ASD, there has been little research that has investigated the efficacy of such stimuli on socio-communicative responsiveness measures. Here, we present preliminary results from a pilot study which tested whether sung instruction, as compared to spoken directives, could elicit greater number of socio-communicative behaviors in young children with ASD. Using an adapted single-subject design, three children between the ages of 3 and 4 years, participated in a programme consisting of 18 sessions, of which 9 were delivered with spoken directives and 9 with sung. Sessions were counterbalanced and randomized for three play activities - block matching, picture matching and clay play. All sessions were video-recorded for post-hoc observational coding of three behavioral metrics which included performance, frequency of social gesture and eye contact. Analysis of the videos by two independent raters indicated increased socio-communicative responsiveness in terms of frequency of social gesture as well as eye contact during sung compared to spoken conditions, across all participants. Our findings suggest that sung directives may play a useful role in engaging children with ASD and also serve as an effective interventional medium to enhance socio-communicative responsiveness.

Keywords: autism, socio-communicative responsiveness, song, joint attention, eye contact

\section{Introduction}

Impairments in the socio-communicative domain are a hallmark feature of Autism Spectrum Disorders (ASD) (Kanner, 1943a; Zwaigenbaum et al., 2005; American Psychiatric Association, 2013). These impairments are reflected in behaviors such as the inability to orient socially, understanding and use of social gestures, gaze following, eye contact, imitation as well as the capacity to initiate and/or respond to joint attention. An extensive body of research has established these early emerging social behaviors as important building blocks for a typical developmental trajectory (Mundy et al., 1990; Charman et al., 2003). More specifically, these behaviors are critical in initiating and maintaining social relationships and verbal language development. A number of 
studies have shown that there are significant challenges in the development of skills associated with these socio-communicative behaviors in children with autism (Mundy et al., 1986; Charman et al., 1997; Dawson et al., 1998, 2004; Lozier et al., 2014). Consequently, such behaviors are important targets for early intervention in children with ASD (Warreyn et al., 2005; Jones et al., 2006; Leekam and Ramsden, 2006; Whalen et al., 2006).

An emerging practice for targeting socio-communicative impairments in ASD is the use of music- and song-based interventions (Lim, 2010; Wan et al., 2011; Simpson et al., 2013). Historically, the use of music has always been associated with increased engagement and a preserved domain of functioning. In the earliest scientific account, Kanner had noted the exceptional musical capacity of children with autism (Kanner, 1943a,b). Subsequent investigations further confirmed that children with autism showed a preference for musical stimuli (Thaut, 1988; Buday, 1995). These were also accompanied by numerous anecdotal reports that described the unique and profound effect music has on children with autism (Sacks, 2007). Other studies of musical abilities have demonstrated enhanced skills such as perfect pitch and good melodic memory in children with ASD (Heaton et al., 2008; Molnar-Szakacs and Heaton, 2012; Ouimet et al., 2012). In the domain of affect and music, Heaton et al. (1998) showed that children with autism had a good understanding of the affective implications of musical mode and were able to pair happy and sad faces with excerpts of music in major and minor keys, suggesting that the inability to identify emotions in social stimuli like faces, did not apply to the musical domain. It is also important to note that musical preferences in individuals with autism develop early in life (Allen et al., 2009) and responsiveness to music is found to remain preserved in adults on the autism spectrum, though it is often underestimated due to their reduced ability to articulate it (Allen et al., 2013). However, integrated reviews of the literature on music therapy (MT) interventions have consistently noted music's potential to support the social and affective development of young children with autism (Whipple, 2004; Kaplan and Steele, 2005; Gold et al., 2006; Accordino et al., 2007; Simpson and Keen, 2011).

Recently, studies from the neuroimaging domain have also provided compelling biological evidence showing preserved neural activity for music processing in children with ASD (Lai et al., 2012; Sharda et al., 2015). For instance, a neuroimaging study by Caria et al. (2011), showed that individuals with ASD recruit regions involved in emotion and reward processing while listening to happy and sad musical excerpts, similar to neurotypical controls. On the other hand, two studies (Lai et al., 2012; Sharda et al., 2015) showed that brain regions that show decreased activation during speech stimulation in ASD vs. controls showed greater activation during song stimulation. In fact, the study by Sharda et al. (2015), also demonstrated that fronto-temporal connectivity in the brain remains intact during perception of sung but not spoken words in children with ASD (Sharda et al., 2015). These findings provide robust neurobiological support for the use of music and song stimuli for therapeutic purposes and suggest that the sung stimulus might be a powerful medium to engage a child with ASD.
Since ASD presents a unique condition where sociocommunicative impairments and enhanced music perceptual abilities coexist, clinicians have often attempted to capitalize on the musical strengths of individuals to compensate for their social difficulties (Alvin, 1978; Alvin and Warwick, 1992; Vaiouli et al., 2015). Recently, MT has been classified as an emerging evidence-based practice, useful in teaching individual skills or goals, through the use of specific musical components, such as songs, rhythm, and movement (Geretsegger et al., 2014; Thaut et al., 2015). Although MT has long been used for rehabilitation of neurological disorders (Wan et al., 2010b) and cognitive development (Paul et al., 2012), its potential and validation to improve social, cognitive and motor skills for individuals with autism (American Music Therapy Association, 1999, 2003; Kaplan and Steele, 2005; Molnar-Szakacs and Heaton, 2012) is still an emerging field. The literature pertaining to the use of music as an interventional medium in ASD has focused predominantly on socio-communicative behaviors, with music being consistently used to explore the development of social skills in children (Duffy and Fuller, 2000; Finnigan and Starr, 2010). More recently, a novel music intervention based on auditory-motor mapping has been developed to aid expressive language development for non-verbal children with ASD (Wan et al., 2011). Another study comparing infant-directed speech with infant-directed song on the levels of engagement and learning outcomes (Simpson et al., 2015), used spoken and sung conditions embedded in a computer-based communication intervention, developed to teach receptive labeling in children with autism. Combined together, the above studies provide both behavioral and neurobiological motivation for use of music, especially song, as an effective tool for improving sociocommunicative responsiveness in individuals with autism (Gold et al., 2006; Simpson et al., 2013; Geretsegger et al., 2014).

Based on this premise, the aim of the current study was to further investigate the effects of singing on sociocommunicative responsiveness in children with ASD. More, specifically, efficacy of sung-directives to improve eye contact and social responsiveness in children with ASD were studied and the potential of intoned vocalizations and singing as an interventional medium, suited for the clinic and easily adaptable for home and classroom settings, was examined. In contrast to previous cross-over or group level designs, we employed an adapted single subject research design to control for within subject variability (Barlow and Hayes, 1979; Barlow and Herson, 1984; Scruggs et al., 1987; Horner et al., 2005; Kennedy, 2005). The main goal was to assess the efficacy of song as a medium of intervention in ASD, given its intrinsic motivational value. We hypothesized that sung instructions may act as a communicative scaffold for children with ASD and consequently be more engaging and elicit greater number of socially responsive behaviors in participants, as compared to spoken directives.

\section{Methods}

\section{Participants}

Three children, all boys (mean age $=3.36$ years, $S D=$ 0.21) participated in this study. All three children were 
diagnosed using the Diagnostic and Statistical Manual of Mental Disorders-5 (DSM 5, American Psychiatric Association, 2013) and International Classification of Diseases-10 (ICD 10, World Health Organization, 1992) criteria by experienced medical professionals. Standard assessment measures including the Social Responsiveness Scale (SRS 2, Constantino and Gruber, 2012), Childhood Autism Rating Scale (CARS II, Schopler et al., 1980), parental reports and direct child observations were used to confirm the diagnoses. The Vineland Adaptive Behavior Scale (VABS II, Sparrow et al., 2005) was administered to assess adaptive behavior and socio-communicative skills. Detailed demographics are provided in Table 1. To be eligible for participation in the study, the participants had to be (1) formally diagnosed with ASD by a practicing physician, (2) chronologically aged between 3 and 5 years, (3) able to participate in the 18 sessions of the research programme, and (4) without any other comorbid neurological or psychiatric diagnosis. The participants were selected based on parental consultation and informed consent procedures approved by the Institutional Ethics Committee. Detailed information for each child is provided below.

\section{Child A}

Child A was 3 years 4 months old when the study commenced. He had a repertoire of few words, such as "hello," names of objects and people which he could use in 2-3 word sentence combinations for social greetings and need-based communication. He was a socially-oriented child with evident joint attention in high to moderate interest activities. He also displayed delayed echolalia and template language, and was quick to adapt to repetitive routines and patterns in play and social interaction. Child A was the higher functioning child amongst the three participants with a CARS score of 41 (mild to moderate) and SRS of 75. He often showed neutral affect and occasionally produced echolalic words or phrases.

\section{Child B}

Child B was 3 years 7 months old at the start of the programme. He had extremely low functional language skills and frequently uttered vocalizations without any apparent communicative intent. Child B had a CARS score of 52 (severe). He displayed low joint attention in social interactions and often avoided eye contact. He used to engage primarily in solitary play. $\mathrm{He}$ had sensory processing difficulties and emotional dysregulation, which often manifested in disruptive behaviors. His awareness of self and others was low, and sitting tolerance and ability to attend to table top activities was also difficult.

\section{Child C}

Child C was 3 years 2 months old when the study started. He was averbal at the beginning of the study and showed minimal to none signs of communicative intent via verbal or vocal modalities. Child $\mathrm{C}$ was severely affected by autism with a CARS score of 53 . He usually maintained a neutral disposition and avoided eye contact. He was reported to be a child who tended to remain in a world of his own and did not show interest in any
TABLE 1 | Behavioral profile and standardized test scores for all participants.

\begin{tabular}{|c|c|c|c|}
\hline & Child A & Child B & Child C \\
\hline \multicolumn{4}{|l|}{ PARTICIPANT DETAILS: } \\
\hline Age (years) & 3.33 & 3.58 & 3.17 \\
\hline Gender & Male & Male & Male \\
\hline \multicolumn{4}{|l|}{ ASSESSMENTS: } \\
\hline \multicolumn{4}{|l|}{ Childhood Autism Rating Scale (CARS) II } \\
\hline T- score & 41 & 52 & 53 \\
\hline Severity group & $\begin{array}{l}\text { Mild to } \\
\text { moderate }\end{array}$ & Severe & Severe \\
\hline \multicolumn{4}{|l|}{ Social Responsiveness Scale (SRS) II } \\
\hline Total T-score & 75 & 89 & 79 \\
\hline Severity group & Moderate & Severe & Severe \\
\hline Social awareness T score & 74 & 83 & 60 \\
\hline Social cognition T score & 73 & 82 & 77 \\
\hline Social communication T score & 74 & 89 & 77 \\
\hline Social motivation T score & 62 & 76 & 66 \\
\hline $\begin{array}{l}\text { Restricted interests and repetitive behavior } \\
\text { T score }\end{array}$ & 82 & 94 & 96 \\
\hline \multicolumn{4}{|l|}{ DSM-5 compatible scores } \\
\hline $\begin{array}{l}\text { Social communication and interaction } \\
\text { T score }\end{array}$ & 73 & 87 & 74 \\
\hline $\begin{array}{l}\text { Restricted interests and repetitive } \\
\text { behavior T score }\end{array}$ & 82 & 94 & 96 \\
\hline \multicolumn{4}{|l|}{ Vineland Adaptive Behavior Scale (VABS) II } \\
\hline Adaptive behavior composite score & 72 & 71 & 67 \\
\hline Adaptive level & $\begin{array}{l}\text { Moderately } \\
\text { low }\end{array}$ & $\begin{array}{l}\text { Moderately } \\
\text { low }\end{array}$ & Low \\
\hline Communication domain score & 76 & 54 & 59 \\
\hline Daily living skills domain score & 75 & 81 & 77 \\
\hline Socialization domain score & 68 & 77 & 66 \\
\hline Motor skills domain score & 82 & 88 & 81 \\
\hline
\end{tabular}

Summary of behavioral profile of the participants.

social activity. His responses could occasionally be elicited in a therapeutic setting by high interest sensory routines.

\section{Procedure}

The study used an adapted single subject research design of AB type (Barlow and Hayes, 1979; Barlow and Herson, 1984; Scruggs et al., 1987; Horner et al., 2005; Kennedy, 2005). In a single subject design participants serve as their own controls; and thus it was preferred for this study to account for the within-subject variability across the two conditions-(A) spoken directives (considered the baseline condition) and (B) sung directives (considered the treatment condition), as individuals with ASD largely vary on their behavioral profiles. This was a "proof of concept" study to test our hypothesis that song may be more efficient than spoken directives to act as a communicative scaffold and enhance socio-communicative responsiveness in young children with ASD.

\section{Programme}

The programme consisted of 18 sessions over a period of 3 months for each child. Each session consisted of a (A) spoken 
or (B) sung condition. Three activities were used for all sessions. Each activity was used in both sung and spoken conditions. Each session was of 3-4 min duration per condition. Similar directives such as "Hello," "Look at me," "Let's match pictures," "Let's play with blocks" etc. were used in all sessions. Both spoken and sung sessions contained similar semantic content and only differed in the intonation of the directives. This was to ensure that any differences in behavior could be attributed to the musical nature of the directives used. Representative spectrograms for sample stimuli [refer to Supplementary Audio Clips S1, S2 for (A) spoken and (B) sung conditions] are attached in Supplementary Figure S1 to further illustrate this. Every child took part in 9 sessions with spoken directives and 9 sessions with sung directives, counterbalanced and randomized for the three play activities such as block matching, picture matching and clay play. All conditions and activities were further randomized to account for day-to-day variability in each child's performance.

The activities were chosen as the preferred play activities as reported by the therapists. The materials used for these play activities consisted of colored wooden blocks of different shapes, picture matching board games and synthetic modeling clay. Each session took place in a secluded room at the intervention clinic with the participant and the trained therapist seated across from each other at a table. A second caregiver videotaped the sessions from an adjacent position to the participant using a video camera and played no role in conducting the sessions. The therapist delivered the spoken and sung directives during the spoken and sung sessions, respectively while engaging the child in play activities. All sessions were video-recorded for posthoc observational coding of three behavioral metrics including performance, frequency of social gesture, and eye contact as described below.

\section{Independent Variables}

The study examined the participant's socio-communicative responsiveness within two experimental conditions: (A) the baseline spoken directive condition and (B) the treatment sung directive condition. In both conditions each participant was presented with bids within a play context by the therapist with the goal of having the child respond in a socially appropriate manner. The participant-therapist interaction took place in the following format: (1) the therapist would greet and/or present a preferred play material and initiate a communicative bid; (2) the participant was expected to respond; and (3) the participant's response, if correct/appropriate, would be reinforced by applause. The only difference between (A) the baseline spoken and (B) the treatment sung condition was the intonation of directives used by the therapist as illustrated in the Supplementary Figure S1; while all other elements of the session such as the semantic content, session structure, and settings were unaltered.

\section{Dependent Variables}

Several dependent variables were operationally defined in order to characterize the participants' response to the experimenter's communicative bids. (1) "Performance" on each session was measured as a percentage of correct responses with respect to the total number of instructional directives presented to the child during that session. This measure was used as a nonsocial measure of responsiveness to assess the participants' overall performance and comprehension abilities associated with each play session. Socio-communicative responsiveness was measured using two distinct behaviors-social gesture and eye contact. (2) "Social gesture" was defined as the child's physical response to social greeting such as "hi five" and was measured as a percentage of instances of such social touch with respect to the total number of opportunities received from the experimenter. (3) "Eye contact" was measured as the percentage of frequency of eye contact made by the child with respect to the total number of occurrences of name calling by the experimenter. All these measures were evaluated using videos for each session by a trained rater using a custom-made rating scheme (Hooker, 2013).

\section{Reliability}

An independent second rater trained in behavioral coding but blind to the purpose of the study rated 30\% of the video recordings. These videotapes were randomly selected and the three different behavioral measures defined above were coded from each video. Cohen's Kappa value was calculated for all three behavioral measures to assess the inter-rater reliability. There was substantial agreement on looking behavior (kappa $=0.69$ ) and social gesture (kappa $=0.70)$ whereas the kappa value on performance (kappa $=0.82)$ represented almost perfect agreement (Viera and Garrett, 2005). Only the behavioral measures recorded by the primary rater were used for data analysis.

\section{Results}

The results of measured behaviors for all three participants in (A) baseline spoken vs. (B) treatment sung conditions are shown in Figure 1. All participants scored higher in the treatment (sung) condition compared to the baseline (spoken) on all measures including performance, social gesture and eye contact. Child A performed much better in sung condition with a mean of $\sim 78 \%$ correct responses to instructional directives when compared to $48 \%$ mean correct responses for spoken sessions. Child B was a little lower on accuracy with a mean of $\sim 52 \%$ in the spoken condition and a mean of $42 \%$ in the sung condition. The performance of Child $\mathrm{C}$ was quite low and comparable across both spoken and sung conditions (with a mean of 33\% in spoken and a mean of $31 \%$ in sung condition).

The data also indicate that there was a trend of enhanced responsiveness to social gestures in the sung condition, as compared to baseline spoken condition, for all three participants. Child A responded to social gestures with a mean of $77 \%$ in spoken and $\sim 89 \%$ in sung conditions. Child B showed ceiling effects with very high level of responses, particularly in this behavioral category, both in spoken (a mean of 91\%) and sung (a mean of $100 \%$ ) conditions. Finally, Child C also showed a similar pattern with lower responses in (mean of $41 \%$ ) spoken conditions compared to sung (mean of 60\%) conditions, although the variability for performance on spoken conditions $(S D=33 \%)$ was very high. 

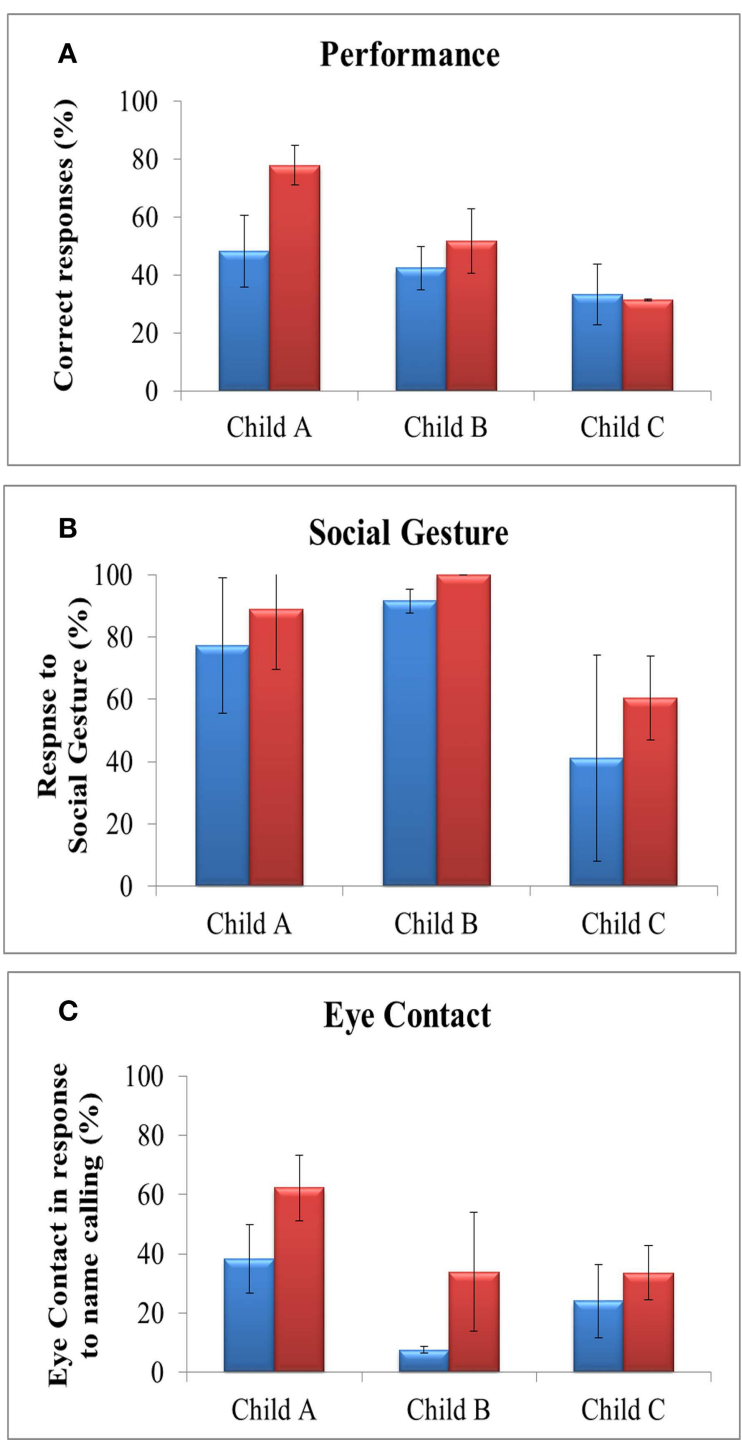

Spoken Sessions $\square$ Sung Sessions

FIGURE 1 | Comparison of behavioral measures in spoken vs. (B) sung sessions. The figure shows the comparison of overall percent scores on the behavioral metrics (A) performance, (B) social gesture, and (C) eye contact for each participant in spoken (blue) vs. sung (red) conditions across all 18 sessions. The means of all three behavioral measures across the sessions revealed an overall increase in sung sessions compared to baseline spoken conditions.

A similar trend of increased frequency of eye contact in response to name calling across the sung sessions was observed. Child A made an average of $38 \%$ eye contact in the spoken sessions compared to a mean of $62 \%$ in sung sessions. Child B responded with a mean of $7.5 \%$ eye contact in spoken condition as compared to a mean of $\sim 34 \%$ in sung condition, though with a high variability $(S D=20)$. Child $C$ also showed an increase in eye contact from a mean of $24 \%$ in spoken condition to a mean of $33 \%$ for sung condition. Overall, the observational analysis of the videos indicated increased socio-communicative responsiveness in terms of both frequency of social gesture as well as eye contact during the sung as compared to the spoken condition, across all 3 participants.

Nevertheless, there was a high degree of variability in the data, revealed by the trajectory of performance for all the participants across 18 sessions, comprising of 9 spoken sessions and 9 sung sessions (Figure 2). Visual inspection was used to examine changes in measured behavior as it is considered to be the most appropriate and most commonly used method of analysis in single-subject design research (Horner et al., 2005; Kennedy, 2005). For all participants, the scores for all measures in the sung sessions were greater than (or equal to) the spoken sessions- performance (Child A-7 out of 9 sessions, Child B-7 out of 9 sessions, Child C-7 out of 9 sessions), social gesture (Child A-8 out of 9, Child B-all sessions, Child C- all sessions), and eye contact (Child A-6 out of 9 sessions, Child B-6 out of 9 sessions, Child C-6 out of 9 sessions). Moreover, the means of all three behavioral measures across the sessions revealed an overall increase in sung sessions compared to baseline spoken conditions (Figure 1).

To further characterize the profile of participants to assess responsiveness to sung vs. spoken directives, VABS socialization and communication domain scores and SRS social communication and interaction (SCI) scores for each child were compared with their overall "responsiveness to sung words" for performance, social gesture and eye contact measures (Figure 3). This measure of responsiveness was calculated as a difference score: $[($ sung - spoken $) /($ sung + spoken $)]$ for all three measures. Child B, with the higher standardized test score in VABS socialization and SRS SCI domains, showed an increased responsiveness to sung directives as reflected by the difference score for socio-communicative responsiveness in comparison with the other two participants. Interestingly, Child $\mathrm{C}$ who had a comparatively lower standardized test scores in VABS socialization and communication and SRS SCI domains also showed comparable responsiveness to sung directives for social gesture, eye contact, and performance.

\section{Discussion}

Findings from the current study indicate the effectiveness of using singing and song-based directives in improving sociocommunicative responsiveness of young children with ASD. Such song-based directives can be implemented as a medium of communication in interventional programmes at home, clinics as well as school-based settings to facilitate communication and interactions between individuals with ASD and their parents and care givers to help build upon their socio-communicative development.

Previous literature on ASD has shown that behaviors such as coordinated eye contact, joint attention (Mundy and Crowson, 1997; Warreyn et al., 2005; Whalen et al., 2006) and dyadic orienting (Leekam and Ramsden, 2006; Koegel et al., 2009a) are important precursors for communication and socialization. In the current study, we were able to evoke social behaviors using sung directives which may serve as a simple albeit effective interventional medium to enhance social interaction and communication in children with ASD. Our findings 


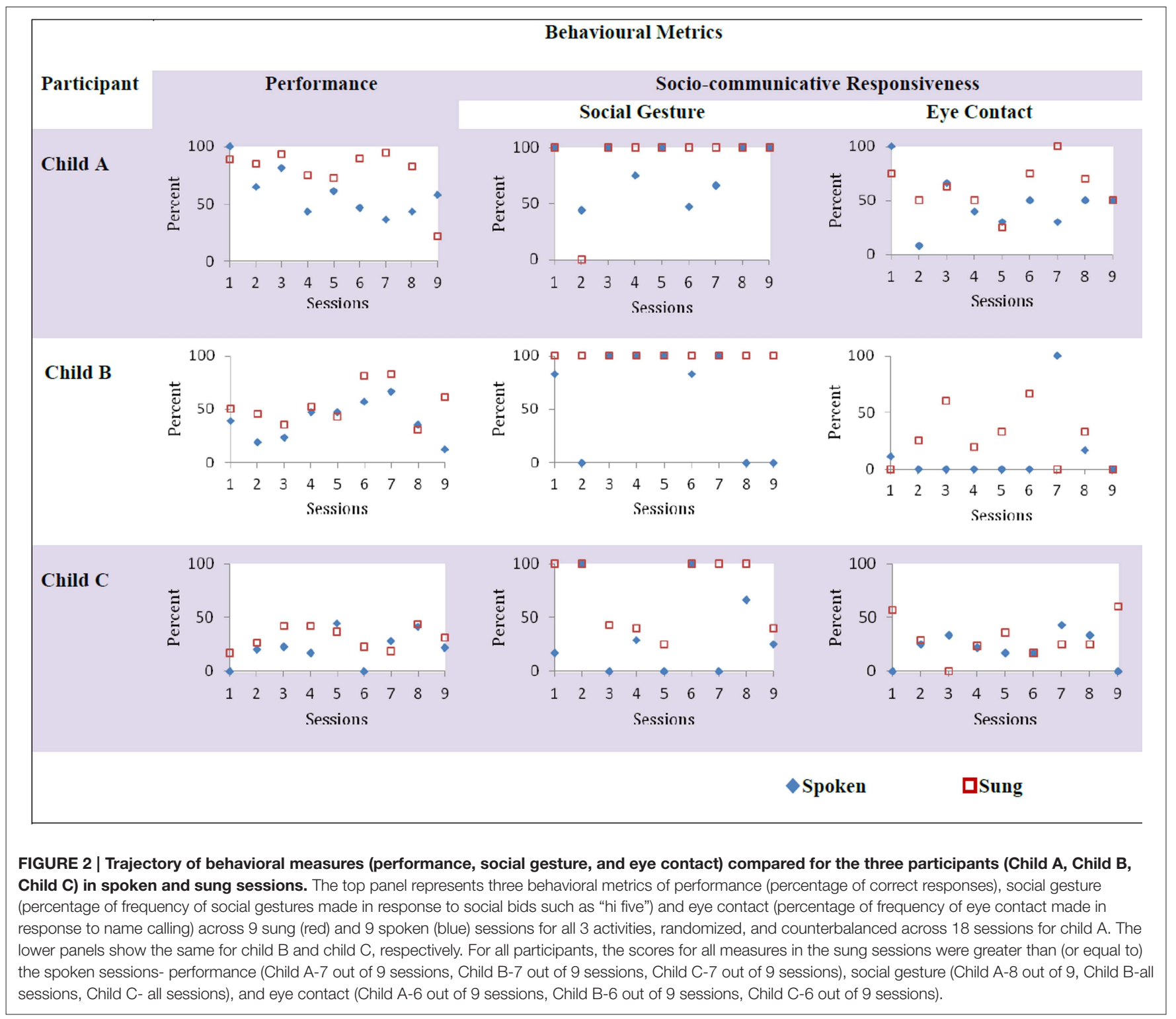

show that singing based directives not only improved sociocommunicative behaviors such as social gesture ("hi five") and eye contact, but also improved non-social behaviors such as performance on a play activity. This suggests that song may not only be engaging, but also provide a communicative scaffold for children with ASD and help in the development of their social skills. This suggests that sung speech may play an important role for children with ASD by engaging them in interactive play activities and increasing attention, compliance, and sociocommunicative skills. The findings from our study corroborate the results obtained in previous research that has used song as a tool for increasing social skills in children with autism (Stevens and Clark, 1969; Buday, 1995; Brownell, 2002; Pasiali, 2004; Kern and Aldridge, 2006; Finnigan and Starr, 2010).

Since ASD is conceptualized largely as a disorder of social impairment leading to delay in communication and other developmental milestones (Garfin and Lord, 1986; Koegel et al.,
1992), most standard therapeutic interventions in ASD, aim at methods to enhance the development of these delayed skills. However, to learn any skill which is not driven by innate motivation, the child is required to engage with the therapist who leads the intervention. This in itself has been and remains an obstacle facing many therapeutic approaches.

Considering the rehabilitative potential of music therapies in facilitating neural plasticity as well as its intrinsic reward value, recent research in neuroscience has provided a robust biomedical perspective for clinical investigation of music therapies in various populations with psychiatric disorders. However, till date there are only few studies which have made an attempt to translate neuroimaging findings in a behavioral context and measure the efficacy of such interventions (e.g., Wan et al., 2010a, 2011; Wan and Schlaug, 2010). For instance, Wan et al. (2011) tested the efficacy of music making on expressive communication in non-verbal children with ASD, using a novel method called 


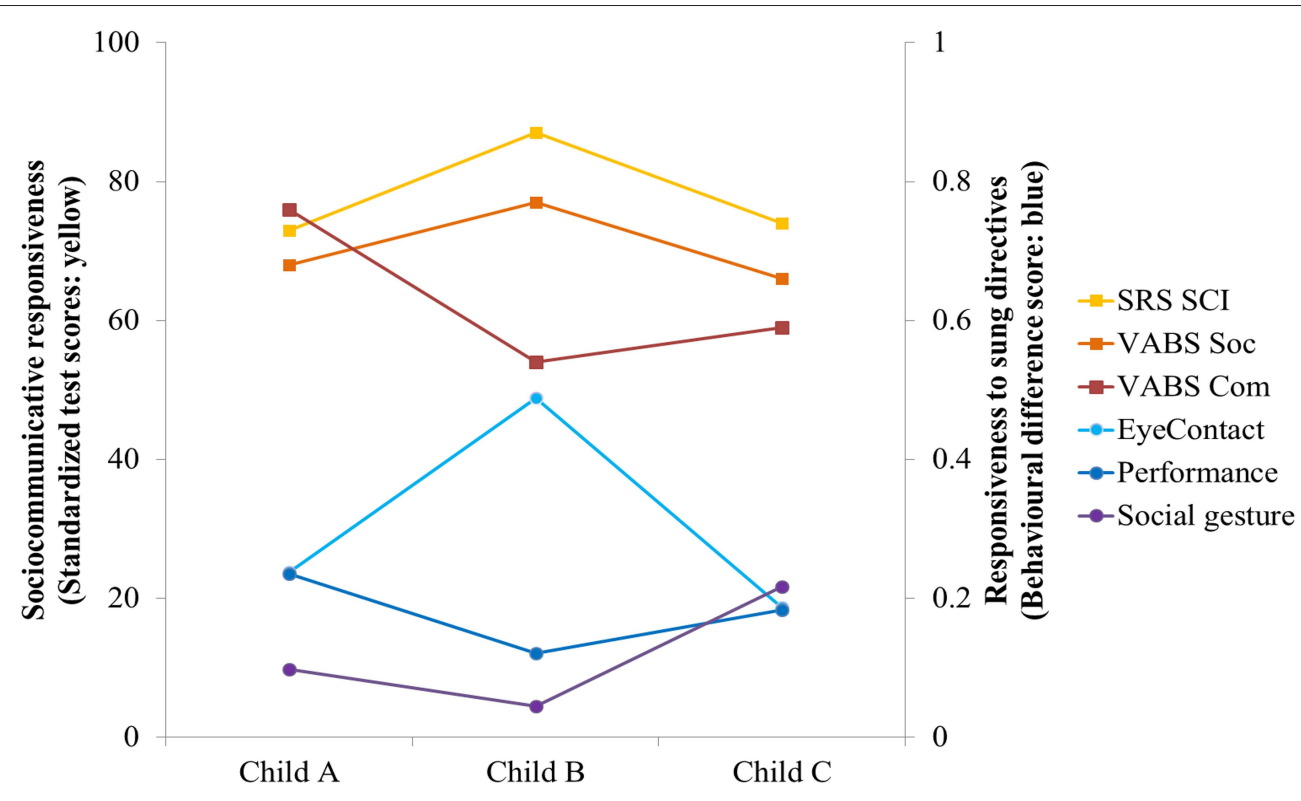

FIGURE 3 | Comparison of responsiveness to sung directives as a function of socio-communicative skills for all participants. The responsiveness to sung directives is defined as the "difference score" [(sung - spoken)/(sung + spoken)]. The difference scores for performance and socio-communicative responsiveness such as social gesture and eye contact (shown in blue) are plotted against socio-communicative skills or standardized test scores such as VABS and SRS (shown in yellow) for all three participants. Child B with higher standardized test score in VABS socialization and SRS SCI domains showed an increased responsiveness to sung directives as reflected by the difference score for socio-communicative responsiveness in comparison with the other two participants. Child $\mathrm{C}$ who had a comparatively lower standardized test scores in VABS socialization and communication and SRS SCI domains also showed comparable responsiveness to sung directives for social gesture, eye contact and performance. VABS, Vineland Adaptive Behavior Scale (subscales-Soc, socialization; Com, communication). SRS, Social Responsiveness Scale (subscales - SCI, social communication and interaction T score; RRB, Restricted Interests and Repetitive Behavior T score).

Auditory-Motor Mapping Training. This was motivated by previous neuroimaging studies that had suggested that the mirror neuron system responsible for imitative behaviors is implicated in ASD (Hadjikhani et al., 2006). In contrast to the Wan et al. (2011) study which focused on expressive communication and speech output in non-verbal children with ASD, our current investigation emphasized on the use of spoken and sung conditions in the receptive domain, particularly on socio-communicative responsiveness, contingent on engagement and motivation of the participants. This study was motivated by recent neuroimaging research which showed that neural pathways are preserved for sung word perception in children with ASD (Sharda et al., 2015) and was a direct follow-up from its findings. As suggested earlier, in another independent study, the neural networks for song processing remain intact and are more effectively engaged in the autistic brain than spoken words (Lai et al., 2012). The findings from our current behavioral study reaffirm such neurophysiological explanations for enhanced behavioral response to sung directives as compared to spoken instructions.

Future studies exploring the potential of song-based interventions could benefit from building upon the findings from this study. Despite the potential of our findings, there were some limitations of this study. Specifically, despite being a powerful design to conduct preliminary studies, a single-case design in which each participant acts as his own control (Barlow and Hayes, 1979; Barlow and Herson, 1984; Scruggs et al.,
1987; Horner et al., 2005; Kennedy, 2005) cannot account for the generalization of results to other settings such as home, classroom or community. Therefore, it is not known whether these improvements would generalize and skills would transfer to other domains, since generalization to a new situation is of particular difficulty for children with autism (Jordan and Powell, 1995). Secondly, there was considerable variability in the data collected for each condition (Figure 1). Consequently, the treatment sung condition did not show any stable trend within the duration of the program, which might be due to the participants' volatility and other factors (Figure 2). Thirdly, since there was no clear order of ability between child A, B, and C, any trends that were observed were hard to interpret and depended on the measure (VABS vs. SRS) used (Figure 3). Therefore, it was difficult to make any generalized conclusions regarding the relationship between overall socio-communicative functioning and responsiveness to sung stimuli. Additionally, child B showed ceiling effects with very high responses particularly in the social gesture behavioral category ( $91 \%$ in spoken and near $100 \%$ in sung conditions), which was reflected in the low difference score of 0.04. A larger sample would help to clarify the situation in future research and lead to more statistically robust findings as indicated in previous music intervention studies (Geretsegger et al., 2014).

Future studies could replicate the current findings using larger samples to establish the validity of song as a therapeutic interventional medium to improve social responsiveness and 
communication. An individualized strategy which uses preferred melody, holds a promising role since preferred activities might be motivating and engaging context for children with autism (Koegel et al., 1987; Koegel and Koegel, 2006). In addition, future studies might also focus on determining which acoustic or musical elements of singing such as pitch, rhythmic pattern or tempo, are most salient in evoking a differential response from the children with ASD. However, the present study provides further empirical support to the anecdotal claims that the children with autism tend to be more engaged by music and songs than speech. Further explorations in this direction would lead to the development of song as a simple and effective interventional tool for children with ASD.

\section{Acknowledgments}

We would like to acknowledge and thank Ms. T. A. Sumathi for assistance in making the spectrographs of speech stimuli and Ms. Sandra Jose for independent rating of the recorded videos.

\section{References}

Accordino, R., Comer, R., and Heller, W. B. (2007). Searching for music's potential: a critical examination of research on music therapy with individuals with autism. Res. Autism Spect. Disord. 1, 101-115. doi: 10.1016/j.rasd.2006.08.002

Allen, R., Davis, R., and Hill, E. (2013). The effects of autism and alexithymia on physiological and verbal responsiveness to music. J. Autism Dev. Disord. 43, 432-444. doi: 10.1007/s10803-012-1587-8

Allen, R., Hill, E., and Heaton, P. (2009). 'Hath charms to soothe...' An exploratory study of how high-functioning adults with ASD experience music. Autism 13, 21-41. doi: 10.1177/1362361307098511

Alvin, J. (1978). Music Therapy for the Autistic Child. Oxford: Oxford University Press.

Alvin, J., and Warwick, A. (1992). Music Therapy for the Autistic Child 2nd Edn. Oxford: Oxford University Press.

American Music Therapy Association (AMTA) (1999). What is Music Therapy? Available online at: http://www.musictherapy.org/quotes.html

American Music Therapy Association (AMTA) (2003). Autism Fact Sheet. Available online at: http://www.musictherapy.org/factsheets/autism.html

American Psychiatric Association (APA) (2013). Diagnostic and Statistical Manual of Mental Disorders, 5th Edn. Washington, DC: American Psychiatric Association (Author).

Barlow, D. H., and Hayes, S. C. (1979). Alternating treatments design: one strategy for comparing the effects of two treatments in a single subject. J. Appl. Behav. Anal. 12, 199-210. doi: 10.1901/jaba.1979.12-199

Barlow, D. H., and Herson, M. (1984). Single Case Experimental Designs: Strategies for Studying Behavior Change. Needham Heights, MA: Allyn \& Bacon.

Brownell, M. K. (2002). Musically adapted social stories to modify behaviours in students with autism: four case studies. J. Music Ther. 39, 117-144. doi: $10.1093 / \mathrm{jmt} / 39.2 .117$

Buday, E. M. (1995). The effects of signed and spoken words taught with music on sign and speech imitation by children with autism. J. Music Ther. 32, 189-202. doi: $10.1093 /$ jmt/32.3.189

Caria, A., Venuti, P., and de Falco, S. (2011). Functional and dysfunctional brain circuits underlying emotional processing of music in autism spectrum disorders. Cereb. Cortex 21, 2838-2849. doi: 10.1093/cercor/bhr084

Charman, T., Baron-Cohen, S., Swettenham, J., Baird, G., Drew, A., and Cox, A. (2003). Predicting language outcome in infants with autism and pervasive developmental disorder. Int. J. Lang. Commun. Disord. 38, 265-285. doi: 10.1080/136820310000104830

Charman, T., Swettenham, J., Baron-Cohen, S., Cox, A., Baird, G., and Drew, A. (1997). Infants with autism: an investigation of empathy, pretend play, joint attention, and imitation. Dev. Psychol. 33, 781-789. doi: 10.1037/00121649.33.5.781
We would like to thank all of the children and parents who participated in this project and the staff and clinicians at Children First Mental Health Institute for assistance in data acquisition. We would also like to thank National Brain Research Centre and Department of Science and Technology for supporting the research project with generous funding.

\section{Supplementary Material}

The Supplementary Material for this article can be found online at: http://journal.frontiersin.org/article/10.3389/fnhum. 2015.00555

Supplementary Figure S1 | Representative spectrograms for spoken vs. sung directives. Spectrographic representations of spoken vs. sung directive ("Hi five!") reflect the similarity of content in overall structure but differences in spectral distribution, in particular, the increased tonality of the sung as compared to the spoken directive.

Supplementary Audio Clips S1, S2 | Representative audio clips for verbal directive in spoken and sung conditions. Audio clips of verbal directive "Hi five!" in spoken condition (S1) and sung condition (S2).

Constantino, J. N., and Gruber, C. P. (2012). Social Responsiveness Scale, $2 n d$ Edn. Los Angeles, CA: Western Psychological Services.

Dawson, G., Meltzoff, A. N., Osterling, J., and Rinaldi, J. (1998). Neuropsychological correlates of early symptoms of autism. Child Dev. 69, 1276-1285. doi: 10.2307/1132265

Dawson, G., Toth, K., Abbott, R., Osterling, J., Munson, J., Estes, A., et al. (2004). Early social attention impairments in autism: social orienting, joint attentions, and attention to distress. Dev. Psychol. 40, 271-283. doi: 10.1037/00121649.40.2.271

Duffy, B., and Fuller, R. (2000). Role of music therapy in social skills development in children with moderate intellectual disability. J. Appl. Res. Intell. Disabil. 13, 77-89. doi: 10.1046/j.1468-3148.2000.00011.x

Finnigan, E., and Starr, E. (2010). Increasing social responsiveness in a child with autism: a comparison of music and non-music interventions. Autism 14, 321-348. doi: 10.1177/1362361309357747

Garfin, D. G., and Lord, C. (1986). "Communication as a social problem in autism," in Social Behavior in Autism, eds E. Schoplet and G. B. Mesibov (New York, NY: Plenum Press), 133-152.

Geretsegger, M., Elefant, C., Mössler, K. A., and Gold, C. (2014). Music therapy for people with autism spectrum disorder. Cochrane Database Syst. Rev. 6:CD004381. doi: 10.1002/14651858.cd004381.pub3

Gold, C., Wigram, T., and Elefant, C. (2006). Music therapy for autistic spectrum disorder. Cochrane Database Syst. Rev. CD004381. doi: 10.1002/14651858.CD004381.pub2

Hadjikhani, N., Joseph, R. M., Snyder, J., and Tager-Flusberg, H. (2006). Anatomical differences in the mirror neuron system and social cognition network in autism. Cereb. Cortex 16, 1276-1282. doi: 10.1093/cercor/bhj069

Heaton, P., Hermelin, B., and Pring, L. (1998). Autism and pitch processing: a precursor for savant musical ability? Music Percept. 15, 291-305.

Heaton, P., Hudry, K., Ludlow, A., and Hill, E. (2008). Superior discrimination of speech pitch and its relationship to verbal ability in autism spectrum disorders. Cogn. Neuropsychol. 25, 771-782. doi: 10.1080/0264329080 2336277

Hooker, J. (2013). Observing Joint Attention in Children with Autism: The Development of a Video Coding Protocol. Undergraduate Honors Theses. Paper 383. Available online at: http://scholar.colorado.edu/honr_theses

Horner, R., Carr, E., Halle, J., McGee, G., Odom, S., and Wolery, M. (2005). The use of single-subject research to identify evidence-based practice in special education. Except. Child. 71, 165-179. doi: 10.1177/001440290507100203

Jones, E. A., Carr, E. G., and Feeley, K. M. (2006). Multiple effects of joint attention intervention for children with autism. Behav. Modif. 30, 782-834. doi: $10.1177 / 0145445506289392$

Jordan, R., and Powell, S. (1995). Understanding and Teaching Children with Autism. Chichester: John Wiley \& Sons Ltd. 
Kanner, L. (1943a). Autistic disturbances of affective contact. Nerv. Child 2, 217-250.

Kanner, L. (1943b). Early infantile autism. J. Pediatr. 25, 211-217.

Kaplan, R. S., and Steele, A. L. (2005). An analysis of music therapy program goals and outcomes for clients with diagnoses on the autism spectrum. J. Music Ther. 42, 2-19. doi: $10.1093 / \mathrm{jmt} / 42.1 .2$

Kennedy, C. H. (2005). Single-case Designs for Educational Research. Boston, MA: Allyn and Bacon.

Kern, P., and Aldridge, D. (2006). Using embedded music therapy interventions to support outdoor play of young children with autism in an inclusive community-based child care program. J. Music Ther. 43, 270-294. doi: $10.1093 / \mathrm{jmt} / 43.4 .270$

Koegel, R. L., and Koegel, L. K. (2006). Pivotal Response Treatments for Autism. Baltimore, MD: Paul H. Brooks Publishing Co.

Koegel, R. L., Dyer, K., and Bell, L. K. (1987). The influence of child-preferred activities on autistic children's social behavior. J. Appl. Behav. Anal. 20, 243-252. doi: 10.1901/jaba.1987.20-243

Koegel, R. L., Koegel, L. K., and Surratt, A. V. (1992). Language intervention and disruptive behavior in preschool children with autism. J. Autism Dev. Disord. 22, 141-153. doi: 10.1007/BF01058147

Koegel, R. L., Vernon, T. W., and Koegel, L. K. (2009a). Improving social initiations in young children with autism using reinforcers with embedded social interactions. J. Autism Dev. Disord. 39, 1240-1251. doi: 10.1007/s10803009-0732-5

Lai, G., Pantazatos, S. P., Schneider, H., and Hirsch, J. (2012). Neural systems for speech and song in autism. Brain 135(Pt 3), 961-975. doi: 10.1093/brain/awr335

Leekam, S. R., and Ramsden, C. A. H. (2006). Dyadic orienting and joint attention in preschool children with autism. J. Autism Dev. Disord. 36, 185-197. doi: 10.1007/s10803-005-0054-1

Lim, H. A. (2010). Effect of "developmental speech and language training through music" on speech production in children with autism spectrum disorders. J. Music Ther. 47, 2-26. doi: 10.1093/jmt/47.1.2

Lozier, L. M., Vanmeter, J. W., and Marsh, A. A. (2014). Impairments in facial affect recognition associated with autism spectrum disorders: a meta-analysis. Dev Psychopathol. 26(4 Pt 1), 933-945. doi: 10.1017/S0954579414000479

Molnar-Szakacs, I., and Heaton, P. (2012). Music: a unique window into the world of autism. Ann. N.Y. Acad. Sci. 1252, 318-324. doi: 10.1111/j.17496632.2012.06465.x

Mundy, P., and Crowson, M. (1997). Joint attention and early social communication: implications for research on intervention with autism. J. Autism Dev. Disord. 27, 653-676. doi: 10.1023/A:1025802832021

Mundy, P., Sigman, M., and Kasari, C. (1990). A longitudinal study of joint attention and language development in autistic children. J. Autism Dev. Disord. 20, 115-128. doi: 10.1007/BF02206861

Mundy, P., Sigman, M., Ungerer, J., and Sherman, T. (1986). Defining the social deficits of autism: the contribution of non-verbal communication measures. J. Child Psychol. Psychiatry Allied Discip. 27, 657-669. doi: 10.1111/j.14697610.1986.tb00190.x

Ouimet, T., Foster, N. E. V., Tryfon, A., and Hyde, K. L. (2012). Auditorymusical processing in autism spectrum disorders: a review of behavioral and brain imaging studies. Ann. N.Y. Acad. Sci. 1252, 325-331. doi: 10.1111/j.17496632.2012.06453.x

Pasiali, V. (2004). The use of prescriptive songs in a home-based environment to promote social skills acquisition by children with autism: three case studies. Music Ther. Perspect. 22, 11-20. doi: 10.1093/mtp/22.1.11

Paul, A., Sharda, M., and Singh, N. C. (2012). Effect of music instruction on cognitive development: a review. J. Indian Inst. Sci. 92, 1-6. Available online at: http://journal.library.iisc.ernet.in/index.php/iisc/article/view/324

Sacks, O. (2007). Musicophilia: Tales of Music and the Brain. New York, NY: Knopf.

Schopler, E., Reichler, R. J., DeVellis, R. F., and Daly, K. (1980). Toward objective classification of childhood autism: Childhood Autism Rating Scale (CARS). J. Autism Dev. Disord. 10, 91-103.

Scruggs, T. E., Mastropieri, M. A., and Casto, G. (1987). The quantitative synthesis of single-subject research: methodology and validation. Remedial Spec. Educ. 8, 24-33. doi: 10.1177/074193258700800206

Sharda, M., Midha, R., Malik, S., Mukerji, S., and Singh, N. C. (2015). Frontotemporal connectivity is preserved during sung but not spoken word listening, across the autism spectrum. Autism Res. 8, 174-186. doi: 10.1002/aur.1437
Simpson, K., and Keen, D. (2011). Music interventions for children with autism: narrative review of the literature. J. Autism Dev. Disord. 41, 1507-1514. doi: 10.1007/s10803-010-1172-y

Simpson, K., Keen, D., and Lamb, J. (2013). The use of music to engage children with autism in a receptive labelling task. Res. Autism Spect. Disord. 7, 1489-1496. doi: 10.1016/j.rasd.2013.08.013

Simpson, K., Keen, D., and Lamb, J. (2015). Teaching receptive labelling to children with autism spectrum disorder: a comparative study using infantdirected song and infant-directed speech. J. Intell. Dev. Disabil. 40, 126-136. doi: 10.3109/13668250.2015.1014026

Sparrow, S. S., Cicchetti, D. V., and Balla, D. A. (2005). Vineland-II Adaptive Behavior Scales: Survey Forms Manual. Circle Pines, MN: AGS Publishing.

Stevens, E., and Clark, F. (1969). Music therapy in the treatment of autistic children. J. Music Ther. 6, 98-104. doi: 10.1093/jmt/6.4.98

Thaut, M. H. (1988). Measuring musical responsiveness in autistic children: a comparative analysis of improvised musical tone sequences of autistic, normal, and mentally retarded individuals. J. Autism Dev. Disord. 18, 561-571. doi: 10.1007/BF02211874

Thaut, M. H., McIntosh, G. C., and Hoemberg, V. (2015). Neurobiological foundations of neurologic music therapy: rhythmic entrainment and the motor system. Front. Psychol. 5:1185. doi: 10.3389/fpsyg.2014.01185

Vaiouli, P., Grimmet, K., and Ruich, L. J. (2015). "Bill is now singing": joint engagement and the emergence of social communication of three young children with autism. Autism 19, 73-83. doi: 10.1177/136236131 3511709

Viera, A. J., and Garrett, J. M. (2005). Understanding interobserver agreement: the kappa statistic. Fam. Med. 37, 360-363.

Wan, C. Y., Bazen, L., Baars, R., Libenson, A., Zipse, L., Zuk, J., et al. (2011). Auditory-motor mapping training as an intervention to facilitate speech output in non-verbal children with autism: a proof of concept study. PLOS ONE 6:e25505. doi: 10.1371/journal.pone.0025505

Wan, C. Y., and Schlaug, G. (2010). Neural pathways for language in autism: the potential for music-based treatments. Future Neurol. 5, 797-805. doi: $10.2217 /$ fnl. 10.55

Wan, C. Y., Demaine, K., Zipse, L., Norton, A., and Schlaug, G. (2010a). From music making to speaking: engaging the mirror neuron system in autism. Brain Res. Bull. 82, 161-168. doi: 10.1016/j.brainresbull.2010.04.010

Wan, C. Y., Ruber, T., Hohmann, A., and Schlaug, G. (2010b). The therapeutic effects of singing in neurological disorders. Music Percept 27, 287-295. doi: 10.1525/mp.2010.27.4.287

Warreyn, P., Roeyers, H., Oelbrandt, T., and De Groote, I. (2005). What are you looking at? Joint attention and visual perspective taking in young children with autism spectrum disorder. J. Dev. Phys. Disabil. 17, 55-73. doi: 10.1007/s10882005-2201-1

Whalen, C., Schreibman, L., and Ingersoll, B. (2006). The collateral effects of joint attention training on social initiations, positive affect, imitation, and spontaneous speech for young children with autism. J. Autism Dev. Disord. 36, 655-664. doi: 10.1007/s10803-006-0108-Z

Whipple, J. (2004). Music in intervention for children and adolescents with autism: a meta-analysis. J. Music Ther. 41, 90-106. doi: 10.1093/ $\mathrm{jmt} / 41.2 .90$

World Health Organization (WHO) (1992). The ICD-10 Classification of Mental and Behavioural Disorders: Clinical Descriptions and Diagnostic Guidelines. Geneva: World Health Organization.

Zwaigenbaum, L., Bryson, S., Rogers, T., Roberts, W., Brian, J., and Szatmari, P. (2005). Behavioral manifestations of autism in the first year of life. Int. J. Dev. Neurosci. 23, 143-152. doi: 10.1016/j.ijdevneu.2004.05.001

Conflict of Interest Statement: The authors declare that the research was conducted in the absence of any commercial or financial relationships that could be construed as a potential conflict of interest.

Copyright (๑ 2015 Paul, Sharda, Menon, Arora, Kansal, Arora and Singh. This is an open-access article distributed under the terms of the Creative Commons Attribution License (CC BY). The use, distribution or reproduction in other forums is permitted, provided the original author(s) or licensor are credited and that the original publication in this journal is cited, in accordance with accepted academic practice. No use, distribution or reproduction is permitted which does not comply with these terms. 